\title{
THE GRADIENT EXPANSION FOR THE FREE-ENERGY OF A CLEAN SUPERCONDUCTOR
}

\author{
ŠIMON KOS, MICHAEL STONE \\ University of Illinois, Department of Physics \\ 1110 W. Green St. \\ Urbana, IL 61801 USA \\ E-mail: m-stone5@uiuc.edu
}

September 5, 2018

\begin{abstract}
We describe a novel method for obtaining the gradient expansion for the free energy of a clean BCS superconductor. We present explicit results up to fourth order in the gradients of the order parameter.
\end{abstract}




\section{Introduction}

The Landau-Ginzburg equations [1] encapsulate much of the physics of conventional superconductors. They were originally proposed on phenomenological grounds, but after the advent of the BCS theory Gor'kov [2] provided a formal derivation of the Landau-Ginzburg free energy, valid in the vicinity of $T_{c}$. Gor'kov's derivation was soon extended to a wider range of temperature by Werthamer [3], and then to higher order in the gradients of the order parameter by Tewordt [4] and Eilenberger [5].

In the approach originally due to Gor'kov and then followed by Eilenberger, Werthamer, Tewordt, and others, the free energy is calculated by relating its variation to the diagonal element of the Green function introduced by Gor'kov [6]. These authors calculated the full Green's function in the Born series, and set its arguments equal at the end. Then they made an ansatz for the free energy in the same approximation by considering all possible terms that may enter with undetermined coefficients. Upon the variation of the ansatz and comparison with the variation of the free energy, they obtained the coefficients. This is very laborious, and moreover nonsystematic.

Since the superconducing gap is much smaller than the Fermi energy, it

is usually safe to linearize the single-particle energy of the normal metal in the vicinity of the Fermi surface. Once this approximation is made, the full Gor'kov Green functions reduce to sums of Green functions, or resolvents, of one-dimensional differential operators [7]. In the years since the publication of the work described above, a great deal has been learned about the properties of such resolvents at points where the arguments coincide. When the Green function concerned is that of a Schrödinger operator, the diagonal element of the resolvent satisfies an equation discovered by Gelfand and Dikii [8]. This allows a fast and efficient evaluation of the diagonal element as an asymptotic expansion in powers of the potential and its derivatives. The terms in this expansion turn out to be the infinite sequence of conserved hamiltonian densities for the Korteweg-de Vries (KdV) hierarchy of integrable partial differential equations [9].

For our superconductivity problem, the one-dimensional Schrödinger operator is replaced by a first-order matrix differential operator of the form studied by Andreev [10. It is identical to a one-dimensional Dirac operator with a chiral mass term. Once again the diagonal element of the resolvent (or a simple modification of this) satisfies an equation which quickly and 
efficiently generates a series expansion in the coeficients of the equation and their derivatives. The terms of this series give the hamiltonian densities for the family of integrable equations known as the AKNS hierarchy [11], whose simplest member is equivalent to the non-linear Schrödinger equation [12]. Perhaps surprisingly, this generating equation has also been exploited in the superconductivity literature. It is nothing other than the Eilenberger equation [13] which plays a central role in the quasiclassical theory [14].

In a previous paper [15] we used both the Gelfand-Dikii equation and the Eilenberger equation to generate the gradient expansion for the free energy as far as the eighth order in the gradients of the gap function. Unfortunately these results are expressed in terms of gradients of the magnitude of the gap-function and gradients of its phase separately. This leads to very long expressions which are of limited utility. We were, however, able to expand the much more compact expressions appearing in the classic paper by Tewordt

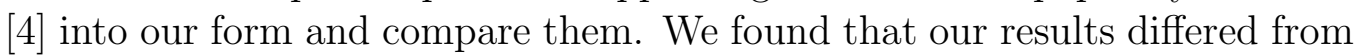
those of Tewordt at the fourth order - the highest order he had computed. While we had confidence in our own results (we had derived them from two completely different methods, using Mathematica to automate the labor), we were not able to isolate the source of the discrepancy.

In this paper we report another method for deriving the gradient expansion. The new method is not quite as efficient at generating the series as that in 15], but has the advantage that the resulting expressions are very compact. We evaluate all terms up to fourth order in the gradients, and expand them out so as to express our results in the form used by Tewordt [四]. We are thus able identify what appears to be a typographical error in his paper. While the correct fourth-order free energy is the principal result of the present paper, we believe that the methods we use are interesting in their own right, and will have application whenever one requires corrections to the leading orders of the quasiclassical method.

In section two we briefly review how the calculation of the free energy of a three dimensional superconductor is reduced to computing determinants of one-dimensional differential operators. In section three we show how the Eilenberger equation arises as a property of the resolvent of such differential operators. In section four we use the Eilenberger function to derive a useful identity linking the dressing function for the Andreev hamiltonian to the determinant we wish to compute. In section five we show how this useful identity is related to the "shooting method" for computing determinants. 
Then, in section six, we use our identity to compute the gradient expansion. Finally in section seven we compare our results with those of reference [4].

\section{From Three Dimensions to One}

In this section we will review standard material so as to establish our notation.

Following Gor'kov [6] we treat the normal metal as a free, highly degenerate, electron gas and the superconductivity as arising from the BCS model potential - an instantaneous short-range attraction between pairs of electrons whose energy is within a narrow shell of width $\omega_{\text {debye }}$ about the Fermi surface. We also ignore paramagnetic effects. The partition function of such an electron gas may be written as a Berezin path integral

$$
\begin{aligned}
& \mathcal{Z}=\operatorname{Tr}\left(e^{-\beta H}\right) \\
& =\int d[\psi] d\left[\psi^{\dagger}\right] \exp -\int_{0}^{\beta} d^{3} x d \tau\left\{\sum_{\alpha=1}^{2} \psi_{\alpha}^{\dagger}\left(\partial_{\tau}-\frac{1}{2 m} \nabla^{2}-\mu\right) \psi_{\alpha}-V \psi_{1}^{\dagger} \psi_{2}^{\dagger} \psi_{2} \psi_{1}\right\} .
\end{aligned}
$$

The indices $\alpha=1,2$ refer to the two components $\uparrow, \downarrow$, of spin. The Grassmannvalued Fermi fields $\psi, \psi^{\dagger}$, are to be taken antiperiodic under $\tau \rightarrow \tau+\beta$.

A positive value for $V$ corresponds to an attractive interaction between the particles. Given an attractive interaction, and a low enough temperature, the system should be unstable with respect to the onset of superconductivity. To detect this instability we introduce the ancillary complex scalar field $\Delta$ which will become the superconducting gap-function. We use it to decouple the interaction by writing

$$
\begin{gathered}
\mathcal{Z}=\int d[\psi] d\left[\psi^{\dagger}\right] d[\Delta] d\left[\Delta^{*}\right] \exp -\int_{0}^{\beta} d^{3} x d \tau\left\{\sum_{\alpha=1}^{2} \psi_{\alpha}^{\dagger}\left(\partial_{\tau}-\frac{1}{2 m} \nabla^{2}-\mu\right) \psi_{\alpha}\right. \\
\left.-\Delta^{*} \psi_{2} \psi_{1}-\Delta \psi_{1}^{\dagger} \psi_{2}^{\dagger}+\frac{1}{V}|\Delta|^{2}\right\}
\end{gathered}
$$

The equation of motion for $\Delta$ shows us that $\Delta \equiv V \psi_{2} \psi_{1}$.

Taking note of the anticommutativity of the Grassmann fields, the quadratic 
form in the exponent can be arranged as a matrix

$$
\left(\psi_{1}^{\dagger}, \psi_{2}\right)\left(\begin{array}{cc}
\partial_{\tau}+\left(-\frac{1}{2 m} \nabla^{2}-\mu\right) & \Delta \\
\Delta^{*} & \partial_{\tau}-\left(-\frac{1}{2 m} \nabla^{2}-\mu\right)
\end{array}\right)\left(\begin{array}{c}
\psi_{1} \\
\psi_{2}^{\dagger}
\end{array}\right) .
$$

We may now integrate out the fermions, obtaining the functional determinant of the Bogoluibov-de Gennes (BdG) operator

$$
\mathcal{B}=\left(\begin{array}{cc}
\partial_{\tau}+\left(-\frac{1}{2 m} \nabla^{2}-\mu\right) & \Delta \\
\Delta^{*} & \partial_{\tau}-\left(-\frac{1}{2 m} \nabla^{2}-\mu\right)
\end{array}\right) .
$$

The BdG operator acts on two-component Nambu spinors obeying $\psi(\mathbf{x}, \tau+$ $\beta)=-\psi(\mathbf{x}, \tau)$.

Provided we can ignore quantum and thermal fluctuations of the gap function $\Delta(x)=|\Delta| e^{i \theta}$, we can perform the integral over $\Delta$ by simply replacing it by its saddle-point value. In this way the euclidean time effective action for a BCS superconductor is given as

$$
\Gamma(\Delta)=-\ln \operatorname{Det} \mathcal{B}+\int d^{3} x d t \frac{1}{V}|\Delta|^{2} .
$$

In this work we will be concerned with the situation where the gap-function $\Delta$ is time-independent. In this case the functional $\Gamma$ reduces to $\beta \mathcal{F}(\Delta)$ where $\mathcal{F}$ is the free energy.

It is not possible to evaluate $\Gamma$ exactly for an arbitrary gap-function, but when $\Delta$ is much smaller than $E_{F}$ and varies slowly on the scale of the Fermi wavelength, then only momenta near the Fermi surface are important. In this case it is reasonable to replace the single-particle energies of the normal metal by the linearized form

$$
\epsilon(\mathbf{k})=v_{F}\left(|\mathbf{k}|-k_{F}\right),
$$

and to approximate $k^{2} d|k|$ by $k_{F}^{2} d|k|$. Having done this, then with no further approximation, we may then use the results from [7] to write

$$
\Gamma(\Delta)=-2 \pi v_{F} N(0) \int \frac{d \Omega_{\mathbf{n}}}{4 \pi} d^{2} x_{\perp} \ln \operatorname{Det}\left(\partial_{\tau}-i v_{F} \sigma_{3}(\mathbf{n} \cdot \nabla)+|\Delta| \sigma_{1} e^{-i \sigma_{3} \theta}\right) .
$$

What is happening in equation (7) requires some explanation. Firstly $N(0)$ is the density of states at the Fermi surface

$$
N(0)=\frac{m k_{F}}{2 \pi^{2}}
$$


the symbol $\mathbf{n}$ denotes a unit vector, and $v_{F}=k_{F} / m$ is the Fermi velocity. Having chosen a point $\mathbf{k}=\mathbf{n} k_{F}$ on the Fermi surface, we decompose the coordinate vector $\mathbf{x}$ into a part parallel to $\mathbf{n}$, and a part perpendicular

$$
\mathbf{x}=x_{\|} \mathbf{n}+\mathbf{x}_{\perp} .
$$

Then, for fixed $\mathbf{x}_{\perp}$, we compute $\ln \operatorname{Det}\left(\partial_{\tau}+\mathcal{H}\right)$ where $\mathcal{H}$ is the one-dimensional Andreev hamiltonian [10

$$
\mathcal{H}=-i \sigma_{3} v_{F}(\mathbf{n} \cdot \nabla)+|\Delta| \sigma_{1} e^{-i \sigma_{3} \theta}=\left[\begin{array}{cc}
-i v_{F}(\mathbf{n} \cdot \nabla) & \Delta \\
\Delta^{*} & +i v_{F}(\mathbf{n} \cdot \nabla)
\end{array}\right] .
$$

This Dirac-like operator appears as an approximation to the full hamiltonian when we restrict our attention to Nambu spinors of the form $\chi(x) \exp i k_{F} \mathbf{n} \cdot \mathbf{x}$, where $\chi(x)$ is slowly varying. Here the spinor $\chi$ and the gap-function $\Delta$ are to be regarded as functions of $x_{\|}$. Notice that the derivative $\mathbf{n} \cdot \nabla$ coincides with $\partial_{x_{\|}}$. Having evaluated $\ln \operatorname{Det}\left(\partial_{\tau}+\mathcal{H}\right)$, we then integrate the result over the family of parallel lines parameterized by $\mathbf{x}_{\perp}$. Finally the $\int \frac{d \Omega_{\mathbf{n}}}{4 \pi}$ performs an average over the directions $\mathbf{n}$.

If we are given an expression for $\ln \operatorname{Det}\left(\partial_{\tau}+\mathcal{H}\right)$ in the form

$$
\ln \operatorname{Det}\left(\partial_{\tau}+\mathcal{H}\right)=\int_{-\infty}^{\infty} F\left(x_{\|}, \mathbf{x}_{\perp}\right) d x_{\|} d t
$$

then the integrations over $x_{\|}$and $\mathbf{x}_{\perp}$ combine to give an integral over every point in three-dimensional space. To illustrate this, consider a timeindependent $\Delta$ at zero temperature. To second order accuracy in the gradient expansion the part of the free energy coming from gradients of the phase of the order parameter is 16]

$$
\int \frac{v_{F}}{8 \pi}((\mathbf{n} \cdot \nabla) \theta)^{2} d x_{\|} d t
$$

Using

$$
\int \frac{d \Omega_{\mathbf{n}}}{4 \pi} n_{i} n_{j}=\frac{1}{3} \delta_{i j}
$$

and remembering that $k_{f}=m v_{F}$, the effective action becomes

$$
\Gamma(\Delta)=\frac{k_{f}^{3}}{3 \pi^{2}} \int \frac{1}{8 m}(\nabla \theta)^{2} d^{3} x d t
$$


This is not unreasonable. Recognising that

$$
\frac{k_{f}^{3}}{3 \pi^{2}}=\rho_{s},
$$

is the number-density of the electrons, and that the superfluid velocity is

$$
\mathbf{v}_{s}=\frac{1}{2 m} \nabla \theta,
$$

we can rewrite (14) as

$$
\Gamma(\Delta)=\int \frac{1}{2} \rho_{s} m v_{s}^{2} d^{3} x d t .
$$

Since everything is time-independent the $t$ integral is trivial, and from $\Gamma(\Delta)=$ $\beta \mathcal{F}(\Delta)$ we have

$$
\mathcal{F}(\Delta)=\int \frac{1}{2} \rho_{s} m v_{s}^{2} d^{3} x .
$$

Thus this part of the free energy coincides with the kinetic energy of the superflow.

\section{The Resolvent Equation}

When $\mathcal{H}$ is time-independent we have

$$
\ln \operatorname{Det}\left(\partial_{\tau}+\mathcal{H}\right)=\sum_{n=-\infty}^{\infty} \ln \operatorname{Det}\left(i \omega_{n}+\mathcal{H}\right),
$$

where $\omega_{n}=2 \pi\left(n+\frac{1}{2}\right) / \beta$, the fermionic Matsubara frequences, are the eigenvalues of $\partial_{\tau}$.

Our task therefore is to obtain the the Fredholm determinant Det $(\mathcal{H}-E)$ involving the Andreev hamiltonian operator

$$
\mathcal{H}=-i \sigma_{3} \partial_{x}+|\Delta| \sigma_{1} e^{-i \sigma_{3} \theta},
$$

and where $E$ is a general complex number. (We have set $v_{F}=1$.) Such determinants are usually evaluated by use of the variational formula

$$
\delta \ln \operatorname{Det}(\mathcal{H}-E)=\operatorname{Tr}\left\{(\mathcal{H}-E)^{-1} \delta(\mathcal{H}-E)\right\},
$$


where $(\mathcal{H}-E)^{-1}$ denotes the Green function, or resolvent,

$$
G_{\alpha \beta}(x, y, E)=\left\langle\alpha, x\left|(\mathcal{H}-E)^{-1}\right| \beta, y\right\rangle .
$$

The Green function can be regarded as an infinite dimensional matrix in the variables $x, y$, and as a $2 \times 2$ matrix in the Nambu spinor space on which the $\sigma_{i}$ matrices act. The notation $\operatorname{Tr} A$ includes an integration over the $x, y$ labels as well as a conventional trace (to be denoted by tr) over the spinor indices.

Provided $E$ is not an eigenvalue of $\mathcal{H}$, the Green function exists and obeys

$$
\left(-i \sigma_{3} \partial_{x}+|\Delta| \sigma_{1} e^{-i \sigma_{3} \theta}-E\right)_{\alpha \gamma} G_{\gamma \beta}(x, y, E)=\delta(x-y) \delta_{\alpha \beta} .
$$

If $\psi_{\alpha}^{L}$ and $\psi_{\alpha}^{R}$ are solutions to the equation

$$
\left(-i \sigma_{3} \partial_{x}+|\Delta| \sigma_{1} e^{-i \sigma_{3} \theta}-E\right) \psi=0,
$$

satisfying suitable boundary conditions to the left and right respectively, then

$$
\begin{aligned}
& G_{\alpha \beta}(x, y, E)=\frac{i}{W} \psi_{\alpha}^{R}(x) \psi_{\beta^{\prime}}^{L}(y)\left(\sigma_{1}\right)_{\beta^{\prime} \beta} \text { for } \quad x>y \\
& =\frac{i}{W} \psi_{\alpha}^{L}(x) \psi_{\beta^{\prime}}^{R}(y)\left(\sigma_{1}\right)_{\beta^{\prime} \beta} \text { for } \quad y>x .
\end{aligned}
$$

Here

$$
W=W\left(\psi^{L}, \psi^{R}\right)=-i\left(\psi^{L}\right)^{T} \sigma_{2} \psi^{R}=\psi_{1}^{R} \psi_{2}^{L}-\psi_{2}^{R} \psi_{1}^{L} .
$$

The expression $W\left(\psi^{L}, \psi^{R}\right)$ is independent of $x$ for any $\psi^{L}, \psi^{R}$ that are both solutions of $(\mathcal{H}-E) \psi=0$. $W$ plays a role analogous to that of the Wronskian in the theory of scalar linear differential equations. In particular $W$ vanishes identically if and only if $\psi^{L}$ and $\psi^{R}$ are linearly dependent. Such linear dependence signals that $E$ is an eigenvalue.

When making the variation $\delta(\mathcal{H}-E)$ in (21) we will only consider changes in $E$ and $\Delta$, so we will require $G(x, y, E)$ only at the points $x=y$. Now strictly speaking, $G(x, x ; E)$ is not well defined, since the jump condition implied by (23) is

$$
G(x, x+\epsilon)-G(x, x-\epsilon)=i \sigma_{3} .
$$

Fortunately this ambiguity does not affect $\delta \ln \operatorname{Det}(\mathcal{H}-E)$. This is because

$$
\operatorname{tr}\left[\sigma_{3} \delta\left(\begin{array}{cc}
-E & \Delta \\
\Delta^{*} & -E
\end{array}\right)\right]=\operatorname{tr} \delta\left(\begin{array}{cc}
-E & \Delta \\
-\Delta^{*} & E
\end{array}\right)=0
$$


We may therefore safely define the symbol $G(x, x, E)$ to mean the average of its two limits [17]

$$
G(x, x) \stackrel{\text { def }}{=} \lim _{\epsilon \rightarrow 0^{+}} \frac{1}{2}(G(x+\epsilon, x)+G(x, x+\epsilon)) .
$$

With this understanding consider the matrix-valued function $g(x)=$ $2 i G(x, x, E) \sigma_{3}$. In components this is

$$
g(x)_{\alpha \beta}=\frac{1}{W}\left(\psi_{\alpha}^{R} \psi_{\beta^{\prime}}^{L}+\psi_{\alpha}^{L} \psi_{\beta^{\prime}}^{R}\right)\left(i \sigma_{2}\right)_{\beta^{\prime} \beta} .
$$

From the equations obeyed by $\psi^{R}$ and $\psi^{L}$ we immediately see that $g$ obeys the equation

$$
\partial_{x} g=[M, g]
$$

where

$$
M=-i \sigma_{3}\left(|\Delta| \sigma_{1} e^{-i \sigma_{3} \theta}-E\right) .
$$

The reason for the appearance of $\sigma_{3}$ in the definition of $g$ is that $\sigma_{3}$ appears in the coefficient of $\partial_{x}$ in the Andreev operator (20). If we pre-multiply the Andreev operator by $-i \sigma_{3}$, it becomes $-\partial_{x}+M$, where the coefficient of $\partial_{x}$ is now proportional to the identity matrix. The Green function for this modified operator is $G(x, y, E) \sigma_{3}$, and $g(x)$ is, up to the factor of $2 i$, the diagonal element of this Green function in $x$ space (it is still a matrix in Nambu spinor space).

The equation (29) can also be written as a commutator

$$
\left[-\partial_{x}+M, g\right]=0
$$

where we regard $g(x)$ as an operator acting on the space of vector valued functions by pointwise multiplication.

In the superconductivity literature (29) is usually called the Eilenberger equation and $g$ the Eilenberger function [13. It lies at the heart of the quasiclassical theory of superconductors 14]. Equation (29) is also the analogue, for matrix first-order differential operators, of the Gelfand-Dikii equation [8] [15]. Like the Gelfand-Dikii equation, it has extensive applications in the theory of integrable dynamical systems [12. Because we wish to stress that (29) is an exact property of the diagonal element of the resolvent of any first-order matrix differential operator of the form $-\partial_{x}+M$ (rather than an 
approximate property of the partially integrated Gor'kov Green function) we will often refer to it as the resolvent equation.

Using the definition of $W$, it is clear that the particular solution of the resolvent equation given by equation (28) satisfies the condition $\operatorname{tr} g=0$. Similar algebra shows that

$$
g^{2} \psi^{L}=\psi^{L}, \quad g^{2} \psi^{R}=\psi^{R}
$$

Since the two solutions are linearly independent and span the space of Nambu spinors at each $x$, this implies $g^{2}=\mathbf{1}$. This condition is sufficient to determine the solution".

Given $g$ as a function of $E$, it is possible to recover $\ln \operatorname{Det}(\mathcal{H}-E)$. We simply observe that

$$
\frac{\partial}{\partial E} \ln \operatorname{Det}(\mathcal{H}-E)=-\int d x \frac{1}{2 i} \operatorname{tr}\left(g \sigma_{3}\right),
$$

and integrate with respect to $E$.

To obtain a gradient expansion of $g$ we introduce a parameter $z$ (which will ultimately be set equal to 1) and rewrite (29) as

$$
\partial_{x} g=[z M, g]
$$

If we seek solutions in the form

$$
g=\sum_{n=0}^{\infty} g_{n} z^{-n},
$$

we obtain the deceptively simple recurrence equation

$$
\partial_{x} g_{n-1}=\left[M, g_{n}\right] .
$$

At first sight this determines $g_{n}$ in terms of a derivative of $g_{n-1}$, so the $n$-th term contains $n$ gradients of the gap parameter.

\footnotetext{
${ }^{1}$ The normalization $g^{2}=\mathbf{1}$ is specific to the resolvent of the Andreev operator on an infinite line, or on a finite interval with self-adjoint boundary conditions at each end. When periodic boundary conditions are imposed on an interval of length $L$, the normalization becomes $g^{2}=-\mathbf{1} \cot ^{2} k L / 2$, where $k$ is the Bloch momentum corresponding to $E$ in the periodically extended problem.
} 
Unfortunately (36) is not quite as innocent as it looks. The $n$-th recurrence relation only immediately determines $g_{n}$ up to the addition of terms that commute with $M$. All is not lost however. Because $M$ is a traceless $2 \times 2$ matrix, the only undetermined term is in fact proportional to $M-$ and it is possible to find this term from the next equation in the series

$$
\partial_{x} g_{n}=\left[M, g_{n+1}\right] .
$$

Taking a trace shows that $\operatorname{tr} M \partial_{x} g_{n}=0$, and this contains the information we need. Using this method we have evaluated the gradient expansion up to eighth order in the gradients [15]. Because extracting the term $\propto M$ requires an explicit parameterization of the space of $2 \times 2$ matrices, the final expressions are given in terms of gradients of $|\Delta|$ and $\nabla \theta$ separately. It is not at all obvious how to assemble these very lengthy expressions into more compact polynomials in derivatives of $\Delta$ itself. In the following sections we will descibe an alternative approach that does not require dissecting $g$ into parts parallel and perpendicular to $M$, and indeed does not require us to find $g$ directly at all.

\section{A Useful Identity}

In what follows we will concentrate on evaluating the determinant in the case where $\mathcal{H}$ is defined on the infinite real line $\mathbf{R}$. For convenience we will assume that all $x$ dependence of the gap function $\Delta(x)$ takes place in a finite region $\Omega_{0}$, and that to the left and right of $\Omega_{0}$ lie regions $\Omega_{L}$ and $\Omega_{R}$, respectively, in which $\Delta$ takes constant values $\Delta_{L}$ and $\Delta_{R}$.

What we do next is motivated by the discussion of first-order matrix differential operators in [12]. We observe that because $g$ is a $2 \times 2$ traceless matrix, it has distinct eigenvalues and is therefore diagonalizable. Because $g$ also obeys the equation $g^{2}=1$, we see that these eigenvalues are \pm 1 . There therefore exists a $2 \times 2$ matrix $\phi(x)$ such that $g(x)$ can be written $g(x)=\phi(x) \sigma_{3} \phi^{-1}(x)$.

As mentioned earlier the resolvent equation can be written 2

$$
\left[-\partial_{x}+M, g\right]=0 \text {. }
$$

\footnotetext{
${ }^{2}$ We hope that it will be clear from the context when a symbol such as $\partial_{x} g$ refers to the derivative $g^{\prime}$, and when $\partial_{x} g$ is an operator acting on functions according to $\left(\partial_{x} g\right) \varphi=$ $g^{\prime} \varphi+g \varphi^{\prime}$.
} 
Substituting $g=\phi \sigma_{3} \phi^{-1}$ into (38) gives

$$
\phi\left[\phi^{-1}\left(-\partial_{x}+M\right) \phi, \sigma_{3}\right] \phi^{-1}=0
$$

and this implies that

$$
\left[\phi^{-1}\left(-\partial_{x}+M\right) \phi, \sigma_{3}\right]=0
$$

Consequently, if we define a matrix $\Lambda$ by

$$
\phi^{-1}\left(-\partial_{x}+M\right) \phi=-\partial_{x}+\Lambda
$$

then $\Lambda \equiv \phi^{-1} M \phi-\phi^{-1} \partial_{x} \phi$ commutes with $\sigma_{3}$ and so must be diagonal. The matrix $\phi(x)$ is sometimes called a dressing function.

We now derive a useful identity that connects $\Lambda$ with the determinant we are wish to compute. We claim that

$$
\begin{aligned}
\operatorname{tr}(g \delta M) & =\delta \operatorname{tr}\left(\Lambda \sigma_{3}\right)-\frac{1}{2} \partial_{x} \operatorname{tr}\left(\phi \sigma_{3} \delta \phi^{-1}-\delta \phi \sigma_{3} \phi^{-1}\right) \\
& \equiv \delta L+\partial_{x} \alpha .
\end{aligned}
$$

(Here and elsewhere $\delta \phi^{-1}$ denotes $\delta\left(\phi^{-1}\right)$ and not $(\delta \phi)^{-1}$.)

We call the two quantities $L$ and $\alpha$ because of the analogy with classical mechanics. In mechanics we have

$$
\sum_{i}\left(-m_{i} \ddot{q}_{i}-\frac{\partial V}{\partial q_{i}}\right) \delta q_{i}=\delta \sum_{i}\left(\frac{1}{2} m_{i} \dot{q}_{i}^{2}-V\right)+\partial_{t}\left(-\sum_{i} m_{i} \dot{q}_{i} \delta q_{i}\right) .
$$

Here the first term on the right-hand side is the variation of lagrangian, while the term in the total derivative generates the symplectic form which determines the hamiltonian structure. The left-hand side contains the functional derivative of the action, and gives the equations of motion via D'Alembert's principle. Dikii [9] exploits this analogy when discussing the stationary equations of the integrable hierarchies.

Notice that the second term on the right hand side of (42) can equally well be written as $-\partial_{x} \operatorname{tr}\left(\phi \sigma_{3} \delta\left(\phi^{-1}\right)\right)$ or as $\partial_{x} \operatorname{tr}\left(\delta \phi \sigma_{3} \phi^{-1}\right)$. This is easily seen by using $\phi \phi^{-1}=\mathbf{1}$.

To establish (42) we rewrite (41) as

$$
\begin{aligned}
-\partial_{x} \phi+M \phi & =\phi \Lambda \\
\partial_{x} \phi^{-1}+\phi^{-1} M & =\Lambda \phi^{-1}
\end{aligned}
$$


From these we have

$$
\begin{aligned}
& A \stackrel{\text { def }}{=}-\partial_{x} \delta \phi+\delta M \phi+M \delta \phi-\delta \phi \Lambda-\phi \delta \Lambda=0 \\
& B \stackrel{\text { def }}{=} \partial_{x} \delta \phi^{-1}+\phi^{-1} \delta M+\delta \phi^{-1} M-\Lambda \delta \phi^{-1}-\delta \Lambda \phi^{-1}=0
\end{aligned}
$$

Thus

$$
0=\operatorname{tr}\left(A \sigma_{3} \phi^{-1}+\phi \sigma_{3} B\right) .
$$

Expanding this out, using the cyclicity of the trace, the equations $\phi^{-1} M=$ $\Lambda \phi^{-1}-\partial_{x} \phi^{-1}$ and $M \phi=\phi \Lambda+\partial_{x} \phi$, together with $g=\phi \sigma_{3} \phi^{-1}$, we find (42).

To see the utility of (42) observe that from $g=2 i G \sigma_{3}$ and $\delta M=$ $-i \sigma_{3} \delta(\mathcal{H}-E)$ we have

$$
\operatorname{Tr}(g \delta M)=\int_{-\infty}^{\infty} d x \operatorname{tr}(g(x) \delta M(x))=2 \operatorname{Tr}(G \delta \mathcal{H})=2 \delta \ln \operatorname{Det}(\mathcal{H}-E) .
$$

Thus, if it is legitimate to ignore the boundary terms coming from the total derivative, we have

$$
\ln \operatorname{Det}(\mathcal{H}-E)=\int_{-\infty}^{\infty} \frac{1}{2} \operatorname{tr}\left(\Lambda \sigma_{3}\right) d x+\text { constant. }
$$

Here the "constant" is a quantity unaffected by local changes in $\Delta(x)$. It will, however, depend on the asymptotic gap functions $\Delta_{L, R}$.

In general the boundary terms cannot be ignored, but it is possible to choose $\phi$ so that they are zero. We have this freedom because requiring that $g(x)=\phi(x) \sigma_{3} \phi^{-1}(x)$ does not uniquely determine $\phi$. Indeed the replacement $\phi \rightarrow \phi \chi$ with $\chi(x)$ any invertable diagonal matrix leaves $g(x)$ unchanged. Such a "gauge transformation" does however alter $\Lambda$. Under the above substitution we have

$$
\Lambda \rightarrow \Lambda-\chi^{-1} \partial_{x} \chi
$$

By making such a gauge transformation we can transfer any of the constributions to $\operatorname{tr}(g \delta M)$ from $\Lambda$ to the total derivative term, or vice versa. Two extreme choices come immediately to mind.

1. Select $\phi$ so that $\Lambda \equiv 0$. In this case all the contributions to the determinant come from the boundary term. 
2. Select $\phi$ so that the boundary terms constribute nothing to the determinant.

The latter choice is possible because the Green function $G(x, y, E)$ is insensitive to the values of $\Delta$ at large distance from $x$ and $y$. We have assumed that there are asymptotic regions $\Omega_{L, R}$ where $\Delta(x)$ becomes independent of $x$. Once $x$ is well inside either of these regions then $g(x)$ will settle down to a constant value depending only on the asymptotic values $\Delta_{L, R}$. We can therefore select a $\phi$ depending only on $\Delta_{L, R}$ to diagonalize this constant matrix. Once we have done this then $\phi( \pm \infty)$ will be unaltered by variations of $\Delta(x)$ taking place in $\Omega_{0}$. The determinant is then obtained entirely from $\Lambda$. This is the route we will take to compute the gradient expansion.

\section{Connection with "Shooting Method"}

Before we derive the gradient expansion, we should point out that our first choice of $\phi$, the one leading to $\Lambda=0$, is equivalent to the so-called "shooting method" for evaluating the determinant 19. This asserts that, in a suitably interpreted sense, the determinant is proportional to the inverse of the transmission coefficient for scattering off the spatially varying $\Delta(x)$. To see this, observe that if we set $\Lambda=0$, so that

$$
\phi^{-1}\left(-\partial_{x}+M\right) \phi=-\partial_{x}+0,
$$

then we are requiring the columns of $\phi$ to be solutions of

$$
\left(-\partial_{x}+M\right) \psi=0
$$

Equivalently

$$
\left(-i \sigma_{3} \partial_{x}+|\Delta| \sigma_{1} e^{-i \sigma_{3} \theta}-E\right) \psi=0 .
$$

A candidate for $\phi$ is therefore

$$
\phi=\left[\begin{array}{cc}
\psi_{1}^{R} & \psi_{1}^{L} \\
\psi_{2}^{R} & \psi_{2}^{L}
\end{array}\right],
$$

where the colums are composed of the $\psi^{L}$ and $\psi^{R}$ solutions used in constructing the Green function. The inverse is

$$
\phi^{-1}=\frac{1}{W}\left[\begin{array}{cc}
\psi_{2}^{L} & -\psi_{1}^{L} \\
-\psi_{2}^{R} & \psi_{1}^{R}
\end{array}\right]
$$


where, as before, $W=W\left(\psi^{L}, \psi^{R}\right)=\psi_{1}^{R} \psi_{2}^{L}-\psi_{2}^{R} \psi_{1}^{L}$.

A short computation shows that $\phi \sigma_{3} \phi^{-1}=g(x)$, so we have guessed correctly.

Now we write down $\psi^{L}$ and $\psi^{R}$ in the asymptotic regions, which is the only place where we will need them. Let us assume that $E$ is real and that $E^{2}>\left|\Delta_{L}\right|^{2},\left|\Delta_{R}\right|^{2}$, so we have scattering solutions.

When $\Delta=|\Delta| e^{i \theta}$ is constant, a plane-wave solution to

$$
\left(-i \sigma_{3} \partial_{x}+|\Delta| \sigma_{1} e^{-i \sigma_{3} \theta}\right) \psi=E \psi
$$

is given by

$$
\psi=u(k, E, \Delta) e^{i k x}=\left[\begin{array}{c}
E+k \\
\Delta
\end{array}\right] e^{i k x} .
$$

Here $E^{2}=k^{2}+|\Delta|^{2}$.

We now introduce transmission and reflection coefficients, $t_{R, L}$ and $r_{R, L}$ and define

$$
\begin{array}{cc}
\psi^{R}(x)=u\left(k, E, \Delta_{L}\right) e^{i k x}+r_{L} u\left(-k, E, \Delta_{L}\right) e^{-i k x} & x \in \Omega_{L} \\
=t_{L} u\left(k, E, \Delta_{R}\right) e^{i k^{\prime} x} & x \in \Omega_{R} \\
\psi^{L}(x)=u\left(-k, E, \Delta_{R}\right) e^{-i k^{\prime} x}+r_{R} u\left(-k^{\prime}, E, \Delta_{L}\right) e^{i k^{\prime} x} & x \in \Omega_{R} \\
=t_{R} u\left(k, E, \Delta_{L}\right) e^{-i k x} & x \in \Omega_{L} .
\end{array}
$$

The apparently perverse appearance of the subscript $L$ on the reflection and transmission coeficients in $\psi^{R}$ (and mutatis mutandis in $\psi^{L}$ ) is supposed to indictate that the incoming wave was incident from the left (right). The wavenumbers $k$ and $k^{\prime}$ are not necessarily equal since we do not assume that $\left|\Delta_{L}\right|=\left|\Delta_{R}\right|$.

Substituting these expressions into $W\left(\psi^{L}, \psi^{R}\right)$ we find that

$$
\begin{aligned}
W\left(\psi^{L}(x), \psi^{R}(x)\right)=-i\left(\psi^{L}\right)^{T} \sigma_{2} \psi^{R} & =-2 k \Delta_{L} t_{R} & & x \in \Omega_{L} \\
& =-2 k^{\prime} \Delta_{R} t_{L} & & x \in \Omega_{R} .
\end{aligned}
$$

Since $W$ is $x$ independent, we must have

$$
k \Delta_{L} t_{R}=k^{\prime} \Delta_{R} t_{L}
$$


This reduces to $t_{L}=t_{R}$ in the particular case that $\Delta_{L}=\Delta_{R}$.

In order to use $\psi^{R}$ and $\psi^{L}$ in the Green function we require that $k, k^{\prime}$, have a positive imaginary part so that $\psi_{R}$ tends to zero as $x \rightarrow+\infty$, and similarly $\psi_{L}$ tends to zero as $x \rightarrow-\infty$. If we assume that both the real part of $E$ and the real part of $k$ are positive (so that $\psi^{R}$ and $\psi^{L}$ correspond to a real scattering process, and $G$ to outgoing waves) then this requires the addition of positive imaginary part to $E$. If we wish to evaluate $G$, and from this $\operatorname{Det}(\mathcal{H}-E)$, for $E$ below the positive real axis then we must replace $k$ by $-k$ in the above wavefunctions, so that the resulting negative imaginary part of $k$ still leads to convergence. As expected, this means that both $G$ and Det $(\mathcal{H}-E)$ have a branch cut discontinuity across the real $E$ axis whenever asymptotic plane-wave solutions are possible.

Now we use these functions to evaluate $\operatorname{tr}\left(\delta \phi \sigma_{3} \phi^{-1}\right)$. Near $x=-\infty$ we have $\psi^{R} \rightarrow u\left(k, E, \Delta_{L}\right) e^{i k x}$ which is large, while $\psi^{L}=t_{R} u\left(k, E, \Delta_{L}\right) e^{-i k x}$ is small. The only expressions occuring in $\operatorname{tr}\left(\delta \phi \sigma_{3} \phi^{-1}\right)$ involve their product which is finite. Varying $\Delta$ in $\Omega_{0}$ changes $t_{R}$, while leaving $u\left(k, E, \Delta_{L}\right)$ etc. unaltered. We find that

$$
\left.\operatorname{tr}\left(\delta \phi \sigma_{3} \phi^{-1}\right)\right|_{x=-\infty}=\frac{\delta t_{R}}{t_{R}}
$$

Similarly

$$
\left.\operatorname{tr}\left(\delta \phi \sigma_{3} \phi^{-1}\right)\right|_{x=+\infty}=-\frac{\delta t_{L}}{t_{L}}
$$

Inserting these results into (42) leads to

$$
\ln \operatorname{Det}(\mathcal{H}-E)=-\ln t+\text { constant }
$$

It does not matter whether we use $t_{L}$ or $t_{R}$ in this formula because the logarithms differ by terms involving $E$ and $\Delta_{R, L}$, and these can be included in the constant. The constants cancel when we consider ratios of determinants of operators with the same asymptotic $\Delta^{\prime}$ 's. In other words, if $\mathcal{H}$ and $\mathcal{H}^{(0)}$ are two hamiltonians with the same asymptotic behaviour, then

$$
\frac{\operatorname{Det}(\mathcal{H}-E)}{\operatorname{Det}\left(\mathcal{H}^{(0)}-E\right)}=\frac{t_{L}^{(0)}(E)}{t_{L}(E)}=\frac{t_{R}^{(0)}(E)}{t_{R}(E)} .
$$

Notice that the (analytic continuation of) $t$ and $r$ become infinite as $E$ approaches the energy of a bound state. The wavefunctions $\psi^{R}$ and $\psi^{L}$ become proportional to one another, and decay exponentially as $|x| \rightarrow \infty$. The 
resultant vanishing of the determinant is consistent with the interpretation of the ratio of the Fredholm determinants as a regularized version of

$$
\prod_{n} \frac{\left(E_{n}-E\right)}{\left(E_{n}^{(0)}-E\right)}
$$

where the $E_{n}$ are the eigenvalues of $\mathcal{H}$, and the $E_{n}^{(0)}$ the eigenvalues of $\mathcal{H}^{(0)}$. For $E$ in the continuous spectrum the zeros and poles coming from the eigenvalues of $\mathcal{H}$ and $\mathcal{H}^{(0)}$ merge to form the branch-cut noted above.

\section{The Gradient Expansion}

To find the recurrence relation for $\phi$ we multiply the matrix $M$ by $z$ as before. At the end of the calculation, we may set $z=1$. Thus again we are looking for a solution of

$$
[-\partial+z M, g]=0
$$

as a power series in $1 / z$. To find the free energy expansion, we do not need $g$ itself but only $\phi$ and $\Lambda$ that satisfy

$$
z M \phi=\phi^{\prime}+\phi z \Lambda
$$

and are given as power series in $1 / z$.

For the homogeneous superconductor, only the zeroth order term in $\Lambda$

will be non-zero. We find it from the zeroth order expression for $g$. Since $g$ is traceless, squares to the identity, and commutes with $M$, we must have

$$
g_{0}=\frac{1}{\zeta} M
$$

where $\zeta$ is defined by $M^{2}=\zeta^{2} 1$. Since

$$
M=\left(\begin{array}{cc}
\omega_{m} & -i \Delta \\
i \Delta^{*} & -\omega_{m}
\end{array}\right)
$$

(we have now replaced $-E$ by the Matsubara frequencies) we have

$$
\zeta=\sqrt{\omega_{m}^{2}+|\Delta(x)|^{2}} .
$$


From (42) and (70) we find

$$
\delta \operatorname{tr}\left(\sigma_{3} \Lambda_{0}\right)=\operatorname{tr}\left(g_{0} \delta M\right)=2 \delta \zeta,
$$

SO

$$
\operatorname{tr}\left(\Lambda_{0} \sigma_{3}\right)=2 \zeta,
$$

and

$$
\Lambda_{0}=\sigma_{3} \zeta .
$$

Hence, we look for $\Lambda$ in the form

$$
\Lambda=\zeta \sigma_{3}+\frac{\Lambda_{1}}{z}+\frac{\Lambda_{2}}{z^{2}}+\ldots
$$

Comparing coefficients of $z^{1}$ in (69), we obtain the equation for the 0 -th order in $\phi$ :

$$
M \phi_{0}=\phi_{0} \zeta \sigma_{3}
$$

To obtain a recurrence relation for higher-order terms in $\phi$, it turns out that $\phi_{0}$ has to be factored out from the expansion, i.e., we look for $\phi$ in the form

$$
\phi=\phi_{0}\left(1+\frac{\phi_{1}}{z}+\frac{\phi_{2}}{z^{2}}+\ldots\right) .
$$

Now we can go on to calculate the first-order terms in (76) and (78) by comparing the coefficients in (69):

$$
M \phi_{0} \phi_{1}=\phi_{0}^{\prime}+\phi_{0} \Lambda_{1}+\phi_{0} \phi_{1} \zeta \sigma_{3}
$$

Multiplying by $\phi_{0}^{-1}$ on the left and using (77), we obtain

$$
\zeta\left[\sigma_{3}, \phi_{1}\right]-\Lambda_{1}=\phi_{0}^{-1} \phi_{0}^{\prime}
$$

In general, comparing the coefficients of $z^{-n}$ in (69), we get the $n$-th order relation

$$
\zeta\left[\sigma_{3}, \phi_{n+1}\right]-\Lambda_{n+1}=\phi_{n}^{\prime}+\phi_{0}^{-1} \phi_{0}^{\prime} \phi_{n}+\phi_{n} \Lambda_{1}+\phi_{n-1} \Lambda_{2}+\ldots+\phi_{1} \Lambda_{n} .
$$

The $(n+1)$ th terms in the expansions of $\phi$ and $\Lambda$ are thus given by all the previous terms. Moreover the first term on the left-hand side of (81) is perpendicular to $\sigma_{3}$, whereas $\Lambda$ contains a multiple of $\sigma_{3}$ and a multiple of 
$\mathbf{1}$, since it commutes with $\sigma_{3}$. In other words, the first term on the left-hand side contains $\sigma_{1}$ and $\sigma_{2}$, whereas the second one contains $\sigma_{3}$ and 1 . Hence, we obtain the $(n+1)$-th terms in the expansion of $\phi$ and $\Lambda$ by calculating the right-hand side of (81) (in which everything is known from the preceding steps in the recursion), and splitting it up into the transverse and longitudinal parts relative to $\sigma_{3}$.

There is a minor hitch: (81) does not determine the part of $\phi_{n+1}$ that commutes with $\sigma_{3}$. Adding a term to $\phi_{n+1}$ that commutes with $\sigma_{3}$ does change the right-hand side of all subsequent recurrence relations. Such a term may, however, always by removed by changing our choice of the $\phi$ that diagonalises $g$. This will change the free energy density by a total derivative of terms local in the gap. It will not affect the total free energy.

We may therefore proceed to find $\phi$ and $\Lambda$. Unfortunately this will lead to the same lengthy expressions we found in [15] by evaluating the recurrence relation for $g$. The problem is that there is no simple way to write the matrix $\phi_{0}^{-1} \phi_{0}{ }^{\prime}$ in terms of $M$ itself. We will therefore have to choose an explicit basis for the matrices and work with components.

After a lot of thought we realized that is far more convenient to replace the arbitrarily chosen matrix $\sigma_{3}$ in the decomposition $g=\phi \sigma_{3} \phi^{-1}$ by the local matrix $M$. To do this we transform

$$
\begin{aligned}
\phi \rightarrow \Phi & \equiv \phi \phi_{0}^{-1}=\mathbf{1}+\frac{\phi_{0} \phi_{1} \phi_{0}^{-1}}{z}+\frac{\phi_{0} \phi_{2} \phi_{0}^{-1}}{z^{2}}+\ldots \\
& \equiv \mathbf{1}+\frac{\Phi_{1}}{z}+\frac{\Phi_{2}}{z^{2}}+\ldots \\
\Lambda \rightarrow \bar{\Lambda} & \equiv \phi_{0} \Lambda \phi_{0}^{-1}=M+\frac{\phi_{0} \Lambda_{1} \phi_{0}^{-1}}{z}+\frac{\phi_{0} \Lambda_{2} \phi_{0}^{-1}}{z^{2}}+\ldots \\
& \equiv M+\frac{\bar{\Lambda}_{1}}{z}+\frac{\bar{\Lambda}_{2}}{z^{2}}+\ldots
\end{aligned}
$$

Then $L \equiv \operatorname{tr}\left(\Lambda \sigma_{3}\right)=\operatorname{tr}(\bar{\Lambda} M / \zeta)$. By using

$$
\phi_{0} \phi_{n}^{\prime} \phi_{0}^{-1}=\Phi_{n}^{\prime}-\phi_{0}^{\prime} \phi_{0}^{-1} \Phi_{n}+\Phi_{n} \phi_{0}^{\prime} \phi_{0}^{-1}
$$

we can rewrite (81) as

$$
\left[M, \Phi_{n+1}\right]-\bar{\Lambda}_{n+1}=\Phi_{n}^{\prime}+\Phi_{n} \phi_{0}^{\prime} \phi_{0}^{-1}+\Phi_{n} \bar{\Lambda}_{1}+\ldots+\Phi_{n} \bar{\Lambda}_{1} .
$$

The advantage of this expression is that the two factors of $\Phi_{n}$ in the second and third terms on the right hand side are both on the left. This 
enables us to get rid of the awkward expression $\phi_{0}^{\prime} \phi_{0}^{-1}$ by factoring out $\Phi_{n}$ and using the recurrence relation for $n=0$

$$
\left[M, \Phi_{1}\right]-\bar{\Lambda}_{1}=\phi_{0}^{\prime} \phi_{0}^{-1}
$$

We obtain

$$
\left[M, \Phi_{n+1}\right]-\bar{\Lambda}_{n+1}=\Phi_{n}^{\prime}+\Phi_{n}\left[M, \Phi_{1}\right]+\Phi_{n-1} \bar{\Lambda}_{2}+\ldots+\Phi_{n} \bar{\Lambda}_{1}
$$

We need, however, to start the recurrence by obtaining $\Phi_{1}$ in some way. For this we can use the Eilenberger equation (68). From (70) and (77), we get the expansion of $g$ in $1 / z$

$$
\begin{aligned}
g & =\phi \sigma_{3} \phi^{-1}=\Phi \frac{M}{\zeta} \Phi^{-1}=\frac{M}{\zeta}+\frac{1}{z}\left[\Phi_{1}, \frac{M}{\zeta}\right]+\ldots \\
& \equiv g_{0}+\frac{g_{1}}{z}+\ldots
\end{aligned}
$$

The coefficient of $z^{0}$ in $(68)$ gives

$$
g_{0}^{\prime}=\left[M, g_{1}\right]
$$

that is,

$$
\left(\frac{M}{\zeta}\right)^{\prime}=\left[M,\left[\Phi_{1}, \frac{M}{\zeta}\right]\right] .
$$

The double commutator of matrices $A, B, C$ can be written as

$$
[A,[B, C]]=\{\{A, B\}, C\}-\{B,\{A, C\}\}
$$

Here the braces denote an anti-commutator. For three traceless $2 \times 2$ matrices this reduces to a form of the vector triple-product identity

$$
[A,[B, C]]=2 \operatorname{tr}(A B) C-2 \operatorname{tr}(A C) B .
$$

We now choose $\Phi_{1}$ with no longitudinal components, i.e. $\operatorname{tr} \Phi_{1}=\operatorname{tr}\left(\Phi_{1} M\right)=$ 0 . Using $\operatorname{tr} M^{2}=2 \zeta^{2}$, we get

$$
\Phi_{1}=-\frac{1}{4} \frac{1}{\zeta}\left(\frac{M}{\zeta}\right)^{\prime} .
$$


We see the natural appearance of $M / \zeta$ as well as of the derivative multiplied by $1 / \zeta$. Hence, it is natural to divide (84) by $\zeta$, and to define

$$
\begin{aligned}
N & \equiv \frac{M}{\zeta} \\
\underline{\bar{\Lambda}} & \equiv \frac{\bar{\Lambda}}{\zeta}=N+\frac{1}{z} \frac{\bar{\Lambda}_{1}}{\zeta}+\frac{1}{z^{2}} \frac{\bar{\Lambda}_{2}}{\zeta}+\ldots \\
& \equiv N+\frac{\bar{\Lambda}_{1}}{z}+\frac{\bar{\Lambda}_{2}}{z^{2}}+\ldots,
\end{aligned}
$$

and

$$
D \equiv \frac{1}{\zeta} \partial
$$

The symbol $D$ still behaves as a derivative in the sense that it is linear, and obeys the Leibnitz rule

$$
D(A B)=(D A) B+A D B .
$$

In terms of these new variables, we can rewrite (84) as

$$
\left[N, \Phi_{n+1}\right]-\underline{\Lambda}_{n+1}=D \Phi_{n}+\Phi_{n}\left[N, \Phi_{1}\right]+\Phi_{n-1} \underline{\Lambda}_{2}+\ldots+\Phi_{1} \underline{\Lambda}_{n}
$$

and

$$
L=\zeta \operatorname{tr}(\underline{\bar{\Lambda}} N) .
$$

The formula (88) leaves undetermined the part of $\Phi_{n+1}$ that commutes with $N$ (just as (81) did not determine the part of $\phi_{n+1}$ that commuted with $\left.\sigma_{3}\right)$. Again it is convenient to choose the $\Phi$ 's purely transverse, i.e.,

$$
\operatorname{tr} \Phi_{k}=\operatorname{tr}\left(\Phi_{k} N\right)=0, \quad \text { for all } k \geq 1
$$

With this choice we get a recurrence relation for the $\Phi$ 's only, and a very simple expression of $L$ in terms of the $\Phi$ 's.

To see how this comes about, let us think of $N$ as being $\sigma_{3}$ (this can always be achieved at a given point by a global rotation which does not change $L$ ), then the $\Phi$ 's contain $\sigma_{1}$ and $\sigma_{2}$, whereas the $\underline{\bar{\Lambda}}$ 's contain 1 and $\sigma_{3}$. Hence, among the terms in (88), $\underline{\Lambda}_{n+1}$ and $\Phi_{n}\left[N, \Phi_{1}\right]$ contain 1 and $\sigma_{3}$ only, $\left[N, \Phi_{n+1}\right]$ and all the products of the type $\Phi_{j} \underline{\Lambda}_{k}$ contain $\sigma_{1}$ and $\sigma_{2}$ only. Finally $D \Phi_{n}$ potentially contains all four matrices. We can separate the transverse and 
the longitudinal part by taking the commutator and anticommutator of $N$ with both sides of (88). From the commutator we get

$$
\left[N,\left[N, \Phi_{n+1}\right]\right]=\left[N, D \Phi_{n}\right]+\left[N, \Phi_{n-1} \underline{\Lambda}_{2}+\ldots+\Phi_{1} \underline{\Lambda}_{n}\right]
$$

Using the double commutator formula (85) and its special case for traceless matrices, this leads to

$$
\left[N,\left[N, \Phi_{n+1}\right]\right]=2 \operatorname{tr}\left(N^{2}\right) \Phi_{n+1}-2 \operatorname{tr}\left(N \Phi_{n+1}\right) N=4 \Phi_{n+1},
$$

where the second term in the middle expression is zero due to transversality. For the last term on the right-hand side of (89) we use the formula

$$
[A, B C]=\{A, B\} C-B\{A, C\}
$$

to get

$$
\left[N, \Phi_{k} \underline{\bar{\Lambda}}_{j}\right]=\left\{N, \Phi_{k}\right\} \underline{\bar{\Lambda}}_{j}-\Phi_{k}\left\{N, \underline{\bar{\Lambda}}_{j}\right\} .
$$

Since $\left\{\sigma_{3}, \sigma_{1,2}\right\}=0$, the first term on the right-hand side of (91) is zero. So we can rewrite (89) as

$$
4 \Phi_{n+1}=\left[N, D \Phi_{n}\right]-\Phi_{n-1}\left\{N, \underline{\bar{\Lambda}}_{2}\right\}-\ldots-\Phi_{1}\left\{N, \underline{\bar{\Lambda}}_{n}\right\}
$$

We see again the usefulness of introducing $N, \underline{\bar{\Lambda}}$, and $D$.

Notice the appearance of the anticommutators $\left\{N, \underline{\bar{\Lambda}}_{j}\right\}$ on the right-hand side of (92). Due to transversality of the $\Phi$ 's, the $\underline{\bar{\Lambda}}$ 's themselves are eliminated. We can relate these anticommutators to the $\Phi$ 's by taking the anticommutator of $N$ with both sides of (88).

$$
\begin{aligned}
-\left\{N, \underline{\Lambda}_{n+1}\right\} & =\left\{N, D \Phi_{n}\right\}+\left\{N, \Phi_{n}\left[N, \Phi_{1}\right]\right\}= \\
& =D\left(\left\{N, \Phi_{n}\right\}\right)-\left\{D N, \Phi_{n}\right\}-\frac{1}{4}\left\{N, \Phi_{n}[N, D N]\right\} .
\end{aligned}
$$

The first term on the right-hand side of (93) is zero just as in (91). In the last term, we use

$$
0=D \mathbf{1}=D\left(N^{2}\right)=N \cdot D N+(D N) N
$$

to see that

$$
[N, D N]=2 N \cdot D N
$$


Then we use the formula

$$
\{A, B C\}=\{A, B\} C-B[A, C]
$$

to get

$$
-\frac{1}{4}\left\{N, \Phi_{n} 2 N \cdot D N\right\}=-\frac{1}{2}\left\{N, \Phi_{n}\right\} N \cdot D N+\frac{1}{2} \Phi_{n}[N, N \cdot D N] .
$$

The first term on the right-hand side of (94) is again zero; for the second one, we need to evaluate

$$
[N, N D N]=N^{2} D N-N(D N) N=D N+N \cdot N \cdot D N=2 D N,
$$

where we used again the anticommutation of $N$ and $D N$. So we can rewrite (93) as

$$
\begin{aligned}
-\left\{N, \underline{\Lambda}_{n+1}\right\} & =-\left\{D N, \Phi_{n}\right\}+\Phi_{n} \cdot D N= \\
& =-(D N) \cdot \Phi_{n} .
\end{aligned}
$$

We introduce the symbol

$$
\mathcal{L}_{k} \equiv\left\{N, \underline{\bar{\Lambda}}_{k}\right\},
$$

and substitute from (95) to (92) to get a recurrence relation for the $\Phi$ 's only:

$$
4 \Phi_{n+1}=\left[N, D \Phi_{n}\right]-\Phi_{n-1}(D N) \Phi_{1}-\ldots-\Phi_{1}(D N) \Phi_{n_{1}} .
$$

We can then simply calculate the terms in the expansion of $\mathcal{L} \equiv\{N, \underline{\bar{\Lambda}}\}$ as

$$
\mathcal{L}_{n}=(D N) \cdot \Phi_{n-1} .
$$

This gives us the desired lagrangian as

$$
L=\frac{\zeta}{2} \operatorname{tr} \mathcal{L} .
$$

The last three equations provide all the machinary needed to compute the first few terms in the expansion of $L$. We start with

$$
\Phi_{1}=-\frac{1}{4} D N
$$


from (86). Then

$$
\begin{aligned}
4 \Phi_{2} & =\left[N, D \Phi_{1}\right]= \\
& =-\frac{1}{4}\left[N, D^{2} N\right]
\end{aligned}
$$

So

$$
\Phi_{2}=-\frac{1}{16}\left[N, D^{2} N\right]
$$

In the third order, we have

$$
\begin{aligned}
4 \Phi_{3} & =\left[N, D \Phi_{2}\right]-\Phi_{1}(D N) \Phi_{1}= \\
& =-\frac{1}{16}\left[N, D\left[N, D^{2} N\right]\right]-\frac{1}{16}(D N)^{3}= \\
& =-\frac{1}{16}\left[N,\left[D N, D^{2} N\right]\right]-\frac{1}{16}\left[N,\left[N, D^{3} N\right]\right]-\frac{1}{16}(D N)^{3}
\end{aligned}
$$

We now use the formula (85) for the double commutator and get

$$
\begin{aligned}
\Phi_{3}= & -\frac{1}{64}\left\{\{N, D N\}, D^{2} N\right\}+\frac{1}{64}\left\{D N,\left\{N, D^{2} N\right\}\right\}- \\
& -\frac{1}{64}\left\{\{N, N\}, D^{3} N\right\}+\frac{1}{64}\left\{N,\left\{N, D^{3} N\right\}\right\}-\frac{1}{64}(D N)^{3} .
\end{aligned}
$$

These expresions can be simplified. We begin by evaluating the anticommutators

$$
\begin{aligned}
\{N, N\} & =2 N^{2}=2 \mathbf{1} \\
\{N, D N\} & =0 \\
\left\{N, D^{2} N\right\} & =D(\{N, D N\})-\{D N, D N\}=-2(D N)^{2} \\
\left\{N, D^{3} N\right\} & =D\left(\left\{N, D^{2} N\right\}\right)-\left\{D N, D^{2} N\right\}=-2 D\left((D N)^{2}\right)-D\left((D N)^{2}\right)= \\
& =-3 D\left((D N)^{2}\right) .
\end{aligned}
$$

All the anticommutators are proportional to the unit matrix (they are squares of traceless $2 \times 2$ matrices), so

$$
\begin{aligned}
\Phi_{3} & =-\frac{1}{32}\left\{D N,(D N)^{2}\right\}-\frac{1}{32}\left\{\mathbf{1}, D^{3} N\right\}-\frac{3}{64}\left\{N, D\left((D N)^{2}\right)\right\}-\frac{1}{64}(D N)^{3}= \\
& =-\frac{5}{64}(D N)^{3}-\frac{1}{16} D^{3} N-\frac{3}{64} N D\left((D N)^{2}\right) .
\end{aligned}
$$


To obtain the gradient expansion of the free energy up to the fourth order, we need to calculate $\mathcal{L}_{2}$ and $\mathcal{L}_{4}$ from (98):

$$
\begin{aligned}
& \mathcal{L}_{2}=(D N) \Phi_{1}=-\frac{1}{4}(D N)^{2} \\
& \mathcal{L}_{4}=(D N) \Phi_{3}=-\frac{5}{64}(D N)^{4}-\frac{1}{16}(D N) \cdot\left(D^{3} N\right)-\frac{3}{64}(D N) \cdot N \cdot D\left((D N)^{2}\right),
\end{aligned}
$$

SO

$$
\begin{aligned}
& L_{2}=-\frac{\zeta}{8} \operatorname{tr}\left((D N)^{2}\right) \\
& L_{4}=-\frac{5}{128} \zeta \operatorname{tr}\left((D N)^{4}\right)-\frac{1}{32} \zeta \operatorname{tr}\left((D N) \cdot\left(D^{3} N\right)\right)-\frac{3}{64} \zeta \operatorname{tr}\left((D N) \cdot N \cdot D\left((D N)^{2}\right)\right) .
\end{aligned}
$$

We can rewrite $L_{4}$ in a more symmetric way by writing

$$
\zeta \operatorname{tr}\left((D N) \cdot\left(D^{3} N\right)\right)=\zeta D\left(\operatorname{tr}\left((D N) \cdot\left(D^{2} N\right)\right)\right)-\zeta \operatorname{tr}\left(\left(D^{2} N\right)^{2}\right) .
$$

The first term is a total derivative $(\zeta D=\partial$ by definition (87)), and is, therefore thrown out. The last term in $L_{4}$ is equal to zero too, since

$$
\operatorname{tr}((D N) \cdot N)=\frac{1}{2} \operatorname{tr}(\{D N, N\})=0
$$

and $D\left((D N)^{2}\right)$ is proportional to the unity matrix. Thus, up to the fourth order in derivatives, including even-order terms only,

$$
L \equiv L_{0}+L_{2}+L_{4}=\zeta \operatorname{tr}\left\{1-\frac{1}{8}(D N)^{2}-\frac{5}{128}(D N)^{4}+\frac{1}{32}\left(D^{2} N\right)^{2}\right\}
$$

\section{The Free Energy}

The equation (100) at the end of the previous section is the principal result of this paper. It contains, in an extremely compact form, all the information

needed to obtain the free energy. Using the results of section 2 this may be written

$$
\mathcal{F}=\int d^{3} x \frac{\left|\mathbf{H}-\mathbf{H}_{a}\right|^{2}}{8 \pi}+\int d^{3} x \frac{|\Delta|^{2}}{V}+2 \pi N_{0} \int \frac{d \Omega_{\mathbf{n}}}{4 \pi} \int d^{2} x_{\perp} \mathcal{F}^{(1 d)},
$$


where $\mathcal{F}^{(1 d)}$ is given in terms of $L$ as

$$
\mathcal{F}^{(1 d)}=-\frac{T}{2} \sum_{\omega_{m}} \int d x_{\|} L
$$

Since $d^{2} x_{\perp} d x_{\|}=d^{3} x$, we can write

$$
\mathcal{F}=\int d^{3} r\left\{\frac{\left|\mathbf{H}-\mathbf{H}_{a}\right|^{2}}{8 \pi}+\frac{|\Delta|^{2}}{V}-\pi T N_{0} \sum_{\omega_{m}} \frac{d \Omega_{\mathbf{n}}}{4 \pi} L\right\} .
$$

Notice that terms with an odd number of derivatives, and hence an odd number of vectors $\mathbf{n}$, drop out when we average over them. This explains why we calculated only even terms in the expansion of $L$.

We have included a magnetic field in (101). In our earlier calculations, we did not mention the coupling of the electrons to the magnetic potential A. However the component $A_{\|}$can always be gauged away along the line $\mathbf{x}_{\perp}=$ const. by absorbing it into $\theta$. There is therefore no loss of generality in our formulae. To insert $\mathbf{A}$ we merely replace our derivative of the complex order parameter by a covariant derivative, and our derivative of the (real) magnitude of the order parameter by a plain gradient.

We would like to compare our calculation with that of reference [4] as this seems to be the only place where the fourth-order terms have been written down explicitly.

The expression given for the free energy in [4] is, in the notation of that paper,

$$
\begin{aligned}
\mathcal{F} & \stackrel{?}{=} \int d^{3} r\left\{\frac{\left|\mathbf{H}-\mathbf{H}_{a}\right|^{2}}{8 \pi}+N_{0} \beta^{-2}\left[\beta^{2} w+\frac{1}{2}\left(v_{F} \beta\right)^{2}\left\{g|\mathbf{O} \chi|^{2}+\frac{1}{6} g^{\prime}\left(\nabla|\chi|^{2}\right)^{2}\right\}+\right.\right. \\
& +\frac{1}{12}\left(v_{F} \beta\right)^{4}\left\{g^{\prime}\left|\mathbf{O}^{2} \chi\right|^{2}+g^{\prime \prime}\left[\frac{1}{2}|\mathbf{O} \chi|^{4}-|\mathbf{O} \chi|^{2}\left(\nabla^{2}|\chi|^{2}\right)+\frac{1}{10}\left(\nabla^{2}|\chi|^{2}\right)^{2}\right]+\right. \\
& \left.\left.\left.+g^{\prime \prime \prime}\left[\frac{1}{4}\left(\nabla^{2}|\chi|^{2}\right)\left(\nabla|\chi|^{2}\right)^{2}-\frac{1}{2}|\mathbf{O} \chi|^{2}\left(\nabla|\chi|^{2}\right)^{2}\right]\right\}\right]\right\} .
\end{aligned}
$$

Here $\chi=\beta \Delta$ is the dimensionless order parameter, and $\mathbf{O}=\nabla-2 i e \mathbf{A}$ is the covariant derivative. The homogeneous part of the free energy comes from $w(|\chi|)$, while $g$ is a function of $|\chi|^{2}$ that we will identify later ( $g$ is not to be confused with the Eilenberger function). The primes on $g$ denote the derivative with respect to $|\chi|^{2}$, i.e.

$$
g^{\prime} \equiv \frac{d g}{d|\chi|^{2}} .
$$


The directional averaging $\int \frac{d \Omega_{\mathbf{n}}}{4 \pi}$ is not stated explicitly in this formula, but is to be understood. In other words the product of two expresions $\mathbf{A B}$ where $\mathbf{A}, \mathbf{B}$ are either of the two vector operators $\mathbf{O}$ or $\nabla$ is defined as

$$
\mathbf{A B}=\frac{1}{3} \delta_{i j} A_{i} B_{j}
$$

and the product of four factors as

$$
\mathbf{A B C D}=\frac{1}{15}\left(\delta_{i j} \delta_{k l}+\delta_{i k} \delta_{l j}+\delta_{i l} \delta_{j k}\right) A_{i} B_{j} C_{k} D_{l}
$$

We have inserted a question mark over the equals sign in (104), because we believe that there is an error in the fourth-order terms.

We will now expand our expression so as to write it in Tewordt's form. During the calculation, we will keep the primes as derivatives, and only at the end we will replace them by the covariant derivatives or gradients according to the rule

$$
\begin{aligned}
\chi^{\prime} & \rightarrow \mathrm{O} \chi \\
\left(|\chi|^{2}\right)^{\prime} & \rightarrow \nabla|\chi|^{2}
\end{aligned}
$$

Note that the prime over the order parameter means $d / d x$, whereas the prime over $g$ means $d / d|\chi|^{2}$.

We introduce the dimensionless quantities

$$
\mathcal{M} \equiv \beta M=\left(\begin{array}{cc}
2 m+1) \pi & -i \chi \\
i \chi^{*} & -(2 m+1) \pi
\end{array}\right)
$$

and

$$
\xi \equiv \beta \zeta=\sqrt{(2 m+1)^{2} \pi^{2}+|\chi|^{2}}
$$

so

$$
N=\frac{\mathcal{M}}{\xi} \text {. }
$$

Since Tewordt writes out the Fermi velocity $v_{F}$ explicitly (in our calculation, we set $v_{F}=1$ ), the dimensionless derivative $D$ equals

$$
D=\frac{v_{F}}{\zeta} \partial=\frac{v_{F} \beta}{\xi} \partial
$$


From (103), the second-order term in $\mathcal{F}$ is

$$
\begin{aligned}
\mathcal{F}_{2} & =\frac{\pi T N_{0}}{8} \int d^{3} r \sum_{\omega_{m}} \zeta \operatorname{tr}(D N)^{2}= \\
& =N_{0} \beta^{-2} \frac{1}{2}\left(v_{F} \beta\right)^{2} \int d^{3} r \sum_{\omega_{m}} \frac{\pi}{4} \xi \frac{1}{\left(v_{F} \beta\right)^{2}} \operatorname{tr}(D N)^{2},
\end{aligned}
$$

where we pulled out the prefactors that appear in the Tewordt formula. Using (110) and (111), we get

$$
\frac{1}{v_{F} \beta} D N=\frac{1}{\xi}\left(\frac{\mathcal{M}}{\xi}\right)^{\prime}=\frac{\mathcal{M}^{\prime}}{\xi^{2}}-\frac{\mathcal{M} \xi^{\prime}}{\xi^{3}} .
$$

To obtain the result in Tewordt's format, we need to trade the derivatives of $\xi$ for the derivatives of $|\chi|^{2}$. From (109), we see that

$$
\xi^{\prime}=\frac{\left(|\chi|^{2}\right)^{\prime}}{2 \xi},
$$

so

$$
\frac{1}{v_{F} \beta} D N=\frac{\mathcal{M}}{\xi^{2}}-\frac{\mathcal{M}\left(|\chi|^{2}\right)^{\prime}}{2 \xi^{4}} .
$$

Hence,

$$
\frac{1}{\left(v_{F} \beta\right)^{2}}(D N)^{2}=\frac{\mathcal{M}^{\prime 2}}{\xi^{4}}-\left\{\mathcal{M}, \mathcal{M}^{\prime}\right\} \frac{\left(|\chi|^{2}\right)^{\prime}}{2 \xi^{6}}+\frac{\mathcal{M}^{2}\left(|\chi|^{2}\right)^{\prime 2}}{4 \xi^{8}} .
$$

From (108) we see

$$
\begin{aligned}
\mathcal{M}^{2} & =\xi^{2} \mathbf{1} \\
\left\{\mathcal{M}, \mathcal{M}^{\prime}\right\} & =(\mathcal{M})^{2^{\prime}}=\left(|\chi|^{2}\right)^{\prime} \mathbf{1} \\
\mathcal{M}^{\prime 2} & =\chi^{\prime} \chi^{*^{\prime}} \mathbf{1},
\end{aligned}
$$

so

$$
\frac{1}{\left(v_{F} \beta\right)^{2}}(D N)^{2}=\left(\frac{\chi^{\prime} \chi^{* \prime}}{\xi^{4}}-\frac{\left(|\chi|^{2}\right)^{2}}{4 \xi^{6}}\right) \mathbf{1} .
$$

We now use (107) to replace the derivatives, and obtain

$$
\mathcal{F}_{2}=N_{0} \beta^{-2} \frac{1}{2}\left(v_{F} \beta\right)^{2} \int d^{3} r \sum_{\omega_{m}} \frac{\pi}{2}\left(\frac{|\mathbf{O} \chi|^{2}}{\xi^{3}}-\frac{\left(\nabla|\chi|^{2}\right)^{2}}{4 \xi^{5}}\right) .
$$


Comparing the first term to (104), we see immediately

$$
g=\frac{\pi}{2} \sum_{\omega_{m}} \frac{1}{\xi^{3}}
$$

We will also need higher derivatives of $g$ with respect to $|\chi|^{2}$. Using (109), we get

$$
\begin{aligned}
g^{\prime} & =-\frac{3}{4} \pi \sum_{\omega_{m}} \frac{1}{\xi^{5}} \\
g^{\prime \prime} & =\frac{15}{8} \pi \sum_{\omega_{m}} \frac{1}{\xi^{7}} \\
g^{\prime \prime \prime} & =-\frac{105}{16} \pi \sum_{\omega_{m}} \frac{1}{\xi^{9}} .
\end{aligned}
$$

Hence,

$$
\mathcal{F}_{2}=N_{0} \beta^{-2} \frac{1}{2}\left(v_{F} \beta\right)^{2} \int d^{3} r\left(g|\mathbf{O} \chi|^{2}+\frac{g^{\prime}}{6}\left(\nabla|\chi|^{2}\right)^{2}\right)
$$

in agreement with (104).

The fourth-order term is equal to

$$
\begin{aligned}
\mathcal{F}_{4} & =-\pi T N_{0} \int d^{3} r \sum_{\omega_{m}} \zeta \operatorname{tr}\left(-\frac{5}{128}(D N)^{4}+\frac{1}{32}\left(D^{2} N\right)^{2}\right)= \\
& =N_{0} \beta^{-2} \frac{\left(v_{F} \beta\right)^{4}}{12} \int d^{3} r \sum_{\omega_{m}} \frac{\xi \pi}{\left(v_{F} \beta\right)^{4}} \operatorname{tr}\left(\frac{15}{32}(D N)^{4}-\frac{3}{8}\left(D^{2} N\right)^{2}\right)
\end{aligned}
$$

We can calculate the first term immediately from (118)

$$
\frac{1}{\left(v_{F} \beta\right)^{4}}(D N)^{4}=\left(\frac{\left(\chi^{\prime} \chi^{* \prime}\right)^{2}}{\xi^{8}}-\frac{\chi^{\prime} \chi^{* \prime}\left(|\chi|^{2}\right)^{\prime 2}}{2 \xi^{10}}+\frac{\left(|\chi|^{2}\right)^{\prime 4}}{16 \xi^{12}}\right) \mathbf{1} \text {. }
$$

To evaluate the second term in (123), we need to go back to (114):

$$
\begin{aligned}
\frac{1}{\left(v_{F} \beta\right)^{4}} D^{2} N & =\frac{1}{\xi}\left(\frac{\mathcal{M}^{\prime}}{\xi^{2}}-\frac{\mathcal{M}\left(|\chi|^{2}\right)^{\prime}}{2 \xi^{4}}\right)^{\prime}= \\
& =\frac{\mathcal{M}^{\prime \prime}}{\xi^{3}}-\frac{3}{2} \frac{\mathcal{M}^{\prime}\left(|\chi|^{2}\right)^{\prime}}{\xi^{5}}+\mathcal{M}\left(\frac{\left(|\chi|^{2}\right)^{\prime 2}}{\xi^{7}}-\frac{\left(|\chi|^{2}\right)^{\prime \prime}}{2 \xi^{5}}\right)
\end{aligned}
$$


(we used again (113) to obtain the second line), so

$$
\begin{aligned}
\frac{1}{\left(v_{F} \beta\right)^{4}}\left(D^{2} N\right)^{2} & =\frac{\mathcal{M}^{\prime \prime 2}}{\xi^{6}}+\frac{9}{4} \frac{\mathcal{M}^{\prime 2}\left(|\chi|^{2}\right)^{\prime 2}}{\xi^{10}}+\mathcal{M}^{2}\left(\frac{\left(|\chi|^{2}\right)^{\prime 2}}{\xi^{7}}-\frac{\left(|\chi|^{2}\right)^{\prime \prime}}{2 \xi^{5}}\right)^{2}- \\
& -\frac{3}{2}\left\{\mathcal{M}^{\prime \prime}, \mathcal{M}^{\prime}\right\} \frac{\left(|\chi|^{2}\right)^{\prime}}{\xi^{8}}-\frac{3}{2}\left\{\mathcal{M}^{\prime}, \mathcal{M}\right\}\left(|\chi|^{2}\right)^{\prime}\left(\frac{\left(|\chi|^{2}\right)^{\prime 2}}{\xi^{12}}-\frac{\left(|\chi|^{2}\right)^{\prime \prime}}{2 \xi^{10}}\right)+ \\
& +\left\{\mathcal{M}^{\prime \prime}, \mathcal{M}\right\}\left(\frac{\left(|\chi|^{2}\right)^{\prime 2}}{\xi^{10}}-\frac{\left(|\chi|^{2}\right)^{\prime \prime}}{2 \xi^{8}}\right)
\end{aligned}
$$

From (108), (115)-(117), we see that

$$
\begin{aligned}
\mathcal{M}^{\prime \prime 2} & =\chi^{\prime \prime} \chi^{* \prime \prime} \mathbf{1} \\
\left\{\mathcal{M}^{\prime \prime}, \mathcal{M}^{\prime}\right\} & =\left(\mathcal{M}^{\prime 2}\right)^{\prime} \mathbf{1} \\
\left\{\mathcal{M}^{\prime \prime}, \mathcal{M}\right\} & =\left\{\mathcal{M}^{\prime}, \mathcal{M}\right\}^{\prime}-2 \mathcal{M}^{\prime 2}=\left(\left(|\chi|^{2}\right)^{\prime \prime}-2 \chi^{\prime} \chi^{* \prime}\right) \mathbf{1}
\end{aligned}
$$

SO

$$
\begin{aligned}
\frac{1}{\left(v_{F} \beta\right)^{4}}\left(D^{2} N\right)^{2} & =\left(\frac{\chi^{\prime \prime} \chi^{* \prime \prime}}{\xi^{6}}+\frac{1}{4} \frac{\chi^{\prime} \chi^{* \prime}\left(|\chi|^{2}\right)^{\prime 2}}{\xi^{1} 0}-\frac{1}{2} \frac{\left(|\chi|^{2}\right)^{\prime 4}}{\xi^{12}}-\frac{\left(|\chi|^{2}\right)^{\prime \prime}}{4 \xi^{8}}-\right. \\
& \left.-\frac{3}{2} \chi^{\prime} \chi^{* \prime} \frac{\left(|\chi|^{2}\right)^{\prime}}{\xi^{8}}+\frac{3}{4} \frac{\left(|\chi|^{2}\right)^{\prime 2}\left(|\chi|^{2}\right)^{\prime \prime}}{\xi^{10}}+\frac{\chi^{\prime} \chi^{* \prime}\left(|\chi|^{2}\right)^{\prime \prime}}{\xi^{8}}\right)
\end{aligned}
$$

Putting the two terms together gives

$$
\begin{aligned}
\mathcal{F}_{4} & =N_{0} \beta^{-2} \frac{\left(v_{F} \beta\right)^{4}}{12} \int d^{3} r \sum_{\omega_{m}} \pi\left(\frac{15}{16} \frac{\chi^{\prime} \chi^{* \prime 2}}{\xi^{7}}-\frac{21}{32} \frac{\chi^{\prime} \chi^{* \prime}\left(|\chi|^{2}\right)^{\prime 2}}{\xi^{9}}+\frac{111}{\frac{\left(6^{2}\right.}{\xi^{11}}}-\right. \\
& \left.-\frac{3}{4} \frac{\chi^{\prime \prime} \chi^{\prime 4}}{\xi^{*}}+\frac{3}{16} \frac{\left(|\chi|^{2}\right)^{\prime \prime 2}}{\xi^{7}}+\frac{9}{8}\left(\chi^{\prime} \chi^{* \prime}\right)^{\prime} \frac{\left(|\chi|^{2}\right)^{\prime}}{\xi^{7}}-\frac{9}{16} \frac{\left(|\chi|^{2}\right)^{\prime 2}\left(|\chi|^{2}\right)^{\prime \prime}}{\xi^{9}}-\frac{3}{4} \frac{\chi^{\prime} \chi^{* \prime}\left(|\chi|^{2}\right)^{\prime \prime}}{\xi^{7}}\right) .
\end{aligned}
$$

The two underlined terms do not appear in Tewordt's formula, so we need to integrate them by parts (again neglecting the boundary terms):

$$
\frac{111}{16^{2}} \frac{\left(|\chi|^{2}\right)^{\prime 4}}{\xi^{11}}=\frac{111}{16 \times 8}\left(|\chi|^{2}\right)^{\prime 3} \frac{1}{\xi^{10}} \frac{\left(|\chi|^{2}\right)^{\prime}}{2 \xi}=
$$




$$
\begin{aligned}
& =-\frac{1}{9} \frac{111}{16 \times 8}\left(|\chi|^{2}\right)^{\prime 3}\left(\frac{1}{\xi^{9}}\right)^{\prime}= \\
& =-\frac{1}{3} \frac{37}{16 \times 8}\left(\left(|\chi|^{2}\right)^{\prime 3} \frac{1}{\xi^{9}}\right)^{\prime}+\frac{37}{16 \times 8} \frac{\left(|\chi|^{2}\right)^{\prime 2}\left(|\chi|^{2}\right)^{\prime \prime}}{\xi^{9}}
\end{aligned}
$$

and

$$
\frac{9}{8}\left(\chi^{\prime} \chi^{* \prime}\right)^{\prime} \frac{\left(|\chi|^{2}\right)^{\prime}}{\xi^{7}}=\frac{9}{8}\left(\chi^{\prime} \chi^{* \prime} \frac{\left(|\chi|^{2}\right)^{\prime}}{\xi^{7}}\right)^{\prime}-\frac{9}{8} \chi^{\prime} \chi^{* \prime} \frac{\left(|\chi|^{2}\right)^{\prime \prime}}{\xi^{7}}+\frac{63}{16} \frac{\chi^{\prime} \chi^{* \prime}\left(|\chi|^{2}\right)^{\prime 2}}{\xi^{9}}
$$

Therefore,

$$
\begin{aligned}
\mathcal{F}_{4} & =N_{0} \beta^{-2} \frac{\left(v_{F} \beta\right)^{4}}{12} \int d^{3} r \sum_{\omega_{m}} \pi\left(\frac{15}{16} \frac{\chi^{\prime} \chi^{* \prime 2}}{\xi^{7}}+\frac{105}{32} \frac{\chi^{\prime} \chi^{* \prime}\left(|\chi|^{2}\right)^{\prime 2}}{\xi^{9}}-\frac{3}{4} \frac{\chi^{\prime \prime} \chi^{* \prime \prime}}{\xi^{5}}+\right. \\
& \left.+\frac{3}{16} \frac{\left(|\chi|^{2}\right)^{\prime \prime 2}}{\xi^{7}}-\frac{35}{16 \times 8} \frac{\left(|\chi|^{2}\right)^{\prime 2}\left(|\chi|^{2}\right)^{\prime \prime}}{\xi^{9}}-\frac{15}{8} \frac{\chi^{\prime} \chi^{* \prime}\left(|\chi|^{2}\right)^{\prime \prime}}{\xi^{7}}\right)
\end{aligned}
$$

Using (121) and introducing the covariant derivatives and gradients, we finally get

$$
\begin{aligned}
\mathcal{F}_{4} & =N_{0} \beta^{-2} \frac{\left(v_{F} \beta\right)^{4}}{12} \int d^{3} r\left(g^{\prime}\left|\mathbf{O}^{2} \chi\right|^{2}+g^{\prime \prime}\left[\frac{1}{2}|\mathbf{O} \chi|^{4}-|\mathbf{O} \chi|^{2}\left(\nabla^{2}|\chi|^{2}\right)+\frac{1}{10}\left(\nabla^{2}|\chi|^{2}\right)^{2}\right]+\right. \\
& \left.+g^{\prime \prime \prime}\left[-\frac{1}{2}|\mathbf{O} \chi|^{2}\left(\nabla|\chi|^{2}\right)^{2}+\frac{1}{24}\left(\nabla^{2}|\chi|^{2}\right)\left(\nabla|\chi|^{2}\right)^{2}\right]\right) .
\end{aligned}
$$

We see that our formula agrees with Tewordt's result except for the prefactor in the last term $(1 / 24$ instead of $1 / 4)$. This is presumably a typographical error. We believe our result is correct, because we had previously obtained it by two other methods.

\section{Discussion}

While principal result of the paper is the fourth-order term in the free energy, as expressed in equations (100) and (125), the methods used to obtain this term are of interest in their own right. The useful identity linking the dressing function $\phi$ with the determinant is one that we have not seen before, and the purely algebraic (requiring no integration) generation of the terms is 
conceptually simpler than other methods of obtaining such series such as those we used in [15. In particular the present algorithm produces very compact expressions, and is applicable to the computation of determinants of any $2 \times 2$ first-order matrix operator.

\section{Acknowledgments}

This work was supported by the National Science Foundation under grant number DMR94-24511 and by the University of Illinois Science and Technology Center for Superconductivity under grant number NSF-DMR-9120000. We would like to thank Ioan Kosztin for valuable converations. MS would like to thank Gerald Dunne for sending him some of his unpublished notes on the Gelfand-Dikii equation for periodic boundary conditions, and David Waxman for e-mail discussions.

\section{References}

[1] See N. R. Werthamer The Ginzburg Landau equations and their extensions in Superconductivity, R. D. Parks ed., (Marcel Decker NY 1969).

[2] L. P. Gor'kov, Zh. Eksp. Teor. Fiz. 361918 (1959); Soviet Physics JETP 91364 (1959).

[3] N. R. Werthamer, Phys. Rev. 132663 (1963).

[4] L. Tewordt, Z. Phys. 190142 (1966)

[5] G. Eilenberger, Z. Phys. 180385 (1964)

[6] L. P. Gor'kov, Zh. Eksp. Teor. Fiz. 34735 (1958); Soviet Physics JETP 7505 (1958).

[7] D. Waxman, Ann. Phys. (NY). 223129 (1993).

[8] I. M. Gelfand, L. A. Dickey, Funkz. Anal. Priloz. 11 no. 211 (1977).

[9] L. A. Dickey, Soliton Equations and Hamiltonian Systems. (World Scientific 1991). 
[10] A. F. Andreev, Zh. Eksp. Teor. Fiz.46 (1964) 1823; Sov. Phys. JETP 19 (1964) 1228.

[11] M. J. Ablowitz D. J. Kaup, A. C. Newell, H. Segur, Phys. Rev. Lett. 31 125 (1973).

[12] See L. A. Dickey, op. cit. chapter 9.

[13] G. Eilenberger, Z. Phys. 214195 (1968)

[14] W. Serene, D. Rainer, Phys. Rep. 10122 (1983).

[15] I. Kosztin, Š. Kos, M. Stone, A. J. Leggett, cond-mat/9803317

[16] M. Stone, Int. Jou. Mod. Phys. B9 (1995) 1359-1373.

[17] J. Feinberg, Phys. Rev. D51 4503 (1995).

[18] A. L. Shelankov, J. Low. Temp. Phys. 6029 (1985).

[19] See for example: D. Waxman, Ann. Phys. (NY). 241285 (1995); Ann. Phys. (NY) 231256 (1994). 